\title{
Niveles de Origen de las Arterias Renales y Mesentérica Superior Respecto a la Columna Vertebral en Individuos Chilenos. Estudio por Tomografía Computarizada Helicoidal
}

\author{
Origin Levels of the Renal and Superior Mesenteric Arteries in Relation to the \\ Vertebral Column in Chilean Individuals. Helicoidal Computed Tomography Study
}

"Olave, E.; ** Puelma, F.; *Henríquez, J.; ${ }^{* *}$ Cruzat, C. \& ** Soto, A.

OLAVE, E.; PUElMA, F.; HENRÍQUEZ, J.; CRUZAT, C. \& SOTO, A. Niveles de origen de las arterias renales y mesentérica superior respecto a la columna vertebral en individuos chilenos. Estudio por tomografía computarizada helicoidal. Int. J. Morphol., 27(2):447-452, 2009.

RESUMEN: El conocimiento de las variaciones arteriales es importante tanto para el diagnóstico como para los procedimientos quirúrgicos a realizar en el abdomen. Esta investigación se realizó con el propósito de determinar a través de angio TAC, los niveles de origen y número de las arterias renales y mesentérica superior, con respecto a los componentes de la columna vertebral. Para ello, utilizamos los registros angiográficos de 31 pacientes, adultos, chilenos, de ambos sexos, en los cuales localizamos los puntos de origen de las arterias mencionadas, para lo cual dividimos la altura de las vértebras en tercios, considerando además al disco intervertebral. En 26 pacientes, la arteria renal en ambos lados se presentó única; en el lado derecho, la arteria renal se presentó doble en 2 personas y en el izquierdo en 3. La arteria renal derecha se originó a nivel de la vértebra L1 en 11 casos; a nivel del disco intervertebral entre L1 y L2 en 7; a nivel de la vértebra L2 en 10; entre L2 y L3 en 2. La arteria renal izquierda se originó a nivel de L1 en 9 casos, entre L1 y L2 en 6, y, de L2 en 12. La arteria mesentérica superior fue única en todos los casos, localizando su nivel de origen siempre craneal al origen de las arterias renales. Se originó a nivel de la vértebra L1 en 16 casos; a nivel de L2 en 8. Se consideraron también niveles más específicos de origen en relación a una subdivisión de las vértebras. Los resultados obtenidos complementarán el conocimiento sobre estas arterias, aportando a la anatomía quirúrgica de la región.

PALABRAS CLAVE: Anatomía; Aorta abdominal; Arterias renales; Arteria mesentérica superior.

\section{INTRODUCCIÓN}

Los actuales procedimientos quirúrgicos que incluyen transplantes, reconstrucciones vasculares y cirugías abdominales en general, requieren de detallados conocimientos de anatomía vascular. Considerando que los vasos sanguíneos son variables, aún cuando existen padrones denominados normales, siempre es necesario revisar la región a intervenir, sobre todo que, en la actualidad, existen diversas técnicas no invasivas para obtener imágenes y determinar previamente las arterias involucradas en determinadas cirugías.

Son numerosos los pacientes que deben ser intervenidos a causa de problemas renales, cuyas arterias son emitidas por la porción abdominal de la aorta, las que se encuentran en estrecha relación con el origen de la arteria mesentérica superior. La literatura consultada muestra que se pueden observar múltiples arterias renales en porcentajes variables: 11,2 \% Wozniak (2000), $18 \%$ Khamanarong et al. (2004), 20\% Dhar \& Lal (2005). Son muchas las descripciones de casos presentados con más de una arteria renal por lado (Bulic et al., 1996; Loukas et al., 2005; Tanyeli et al., 2006; Deepthinath, 2006; Olave et al., 2007).

En relación al nivel de origen, se han reportado orígenes altos de la arteria renal, desde la aorta como de alguna de sus ramas (Garti \& Meiraz, 1980). Dada la particular relación de las arterias renales (AR) con la arteria mesentérica superior (AMS) y tomando como punto de referencia a los componentes de la columna vertebral, nos propusimos estu-

\footnotetext{
Facultad de Medicina, Universidad de La Frontera, Chile

** Facultad de Ciencias de la Salud, Universidad Católica del Maule, Chile
} 
diar a través de tomografía computarizada helicoidal multidetectores, el nivel de origen de estas arterias, así como determinar el número de AR, relacionándolas con las vértebras y discos intervertebrales de la parte baja de la columna torácica y de la parte lumbar.

\section{MATERIAL Y MÉTODO}

Para este estudio utilizamos los registros angiográficos de 31 pacientes, chilenos, adultos, de ambos sexos, con edades comprendidas entre 25 y 84 años, registros que fueron obtenidos a través de tomografía computarizada helicoidal multidetectores. Las variables consideradas fueron: número, origen y relaciones de las arterias renales y mesentérica superior con los componentes de la columna vertebral. Para el caso de las AR, consideramos los lados derecho e izquierdo de cada paciente. La relación con la columna fue establecida, considerando una división de los cuerpos vertebrales en tres partes, superior, media e inferior, así como también se incluyó a los discos intervertebrales para la referida relación.

\section{RESULTADOS}

La arteria mesentérica superior se encontró única en el $100 \%$ de los casos, localizando su origen siempre craneal a las arterias renales. Su origen en relación a los cuerpos vertebrales se encuentra expresado en la Tabla I.

Tabla I. Niveles de origen de la arteria mesentérica superior respecto a la columna vertebral.

\begin{tabular}{llcc}
\hline Nivel columna vertebral & & $\mathrm{N}^{\circ}$ casos & $\%$ \\
\hline T12 & 1/3 inferior & 1 & 3,2 \\
& Disco T12-L1 & 3 & 9,7 \\
L1 & 1/3 superior & 3 & 9,7 \\
& 1/3 medio & 6 & 19,4 \\
& 1/3 inferior & 7 & 22,6 \\
& Disco L1-L2 & 3 & 9,7 \\
L2 & 1/3 superior & 2 & 6,4 \\
& 1/3 medio & 4 & 12,9 \\
& $1 / 3$ inferior & 2 & 6,4 \\
\hline Total & & 31 & 100 \\
\hline
\end{tabular}

Cada uno de los registros fue esquematizado y se fotografiaron los más representativos.

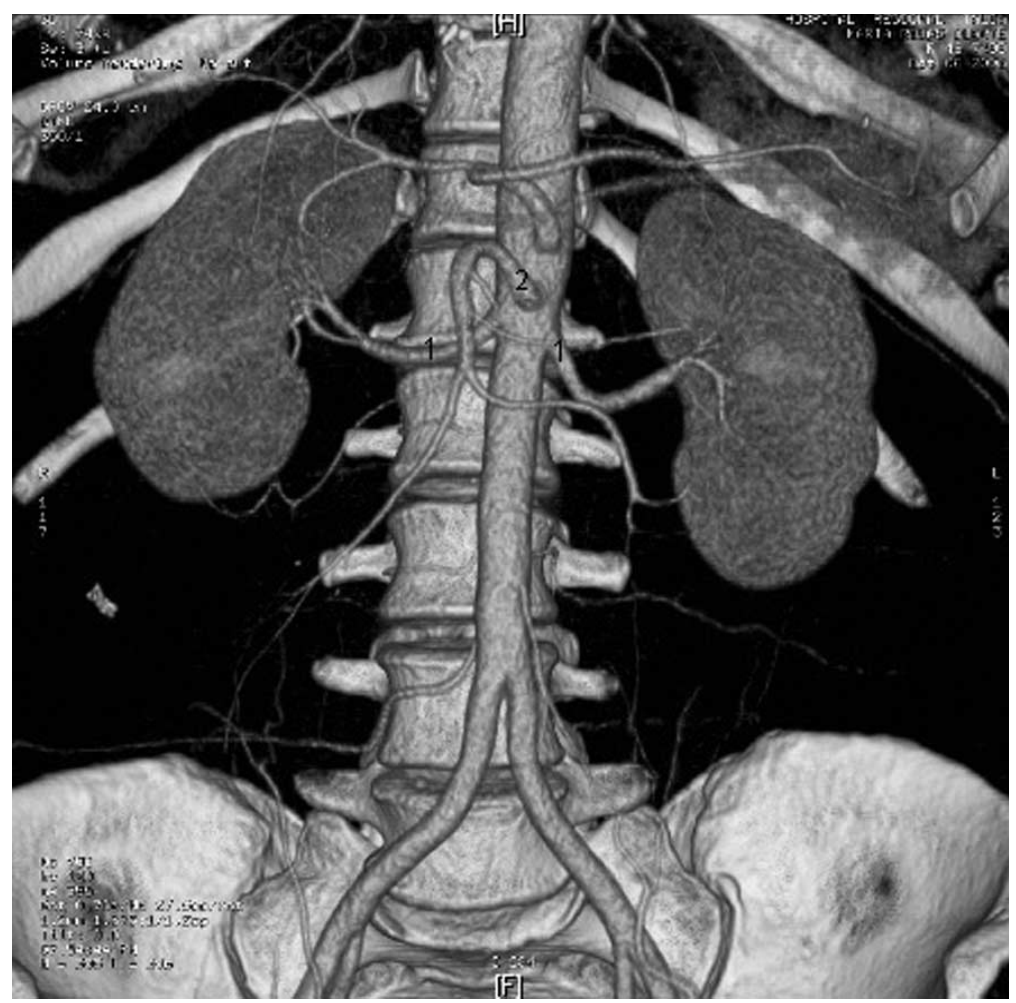

Fig.1. Arterias renales únicas, originándose a nivel del tercio inferior de L1 y arteria mesentérica superior a nivel del tercio medio de la misma vértebra. 1. Arteria renal; 2. Arteria mesentérica superior.
En 26 pacientes $(83,9 \%)$, la arteria renal de ambos lados se presentó única (Figs. 1 y 2); en los restantes 5 pacientes $(16,1 \%)$ se observaron ARR dobles unilaterales (Fig. 3), de las cuales, en 2 pacientes se presentaron en el lado derecho y en 3 , en el izquierdo.

Los niveles de origen de las arterias renales respecto a la columna vertebral son expresados en la Tabla II.

En cuanto a la simetría de origen, de los 26 pacientes con arteria renal única, en $12(46,2 \%)$ el origen de estos vasos fue simétrico y en $14(53,8 \%)$ fue asimétrico. De estos últimos, la arteria derecha tuvo un origen más alto en 8 y la izquierda en 6.

De estos pacientes con más de una arteria, 2 de ellos con arterias dobles en el lado derecho, el origen en este lado y en el izquierdo ocurrió a nivel del tercio medio de la vértebra L1. De los 3 que tuvieron arterias dobles en el lado izquierdo, en 2 de ellos la arteria renal derecha se originó al mismo nivel que la arteria renal superior izquierda (en uno, a nivel del 1/3 inferior de la vértebra L2 y en 
OLAVE, E.; PUELMA, F.; HENRÍQUEZ, J.; CRUZAT, C. \& SOTO, A. Niveles de origen de las arterias renales y mesentérica superior respecto a la columna vertebral en individuos chilenos. Estudio por tomografía computarizada helicoidal. Int. J. Morphol., 27(2):447-452, 2009.

Tabla II. Niveles de origen de las arterias renales en relación a la columna vertebral.

\begin{tabular}{llcccc}
\hline \multicolumn{1}{c}{$\begin{array}{c}\text { Nivel columna } \\
\text { vertebral }\end{array}$} & \multicolumn{2}{c}{ Arteria renal derecha } & \multicolumn{2}{c}{ Arteria renal izquierda } \\
\hline L1 & 1/3 medio & 4 & 12,9 & 4 & 12,9 \\
& $1 / 3$ inferior & 7 & 22,6 & 5 & 16,1 \\
Disco L1-L2 & & 7 & 22,6 & 6 & 19,4 \\
L2 & 1/3 superior & 2 & 6,4 & 4 & 12,9 \\
& $1 / 3$ medio & 4 & 12,9 & 2 & 6,4 \\
& $1 / 3$ inferior & 4 & 12,9 & 6 & 19,4 \\
Disco L2-L3 & & 2 & 6,4 & 2 & 6,4 \\
L3 & $1 / 3$ superior & 1 & 3,2 & 1 & 3,2 \\
L4 & $1 / 3$ superior & 0 & 0,0 & 1 & 3,2 \\
\hline Total & & 31 & 100 & 31 & 100 \\
\hline
\end{tabular}

En el caso de arterias dobles, se consideró la arteria de mayor calibre ( 2 casos en lado derecho y 3 en el izquierdo).

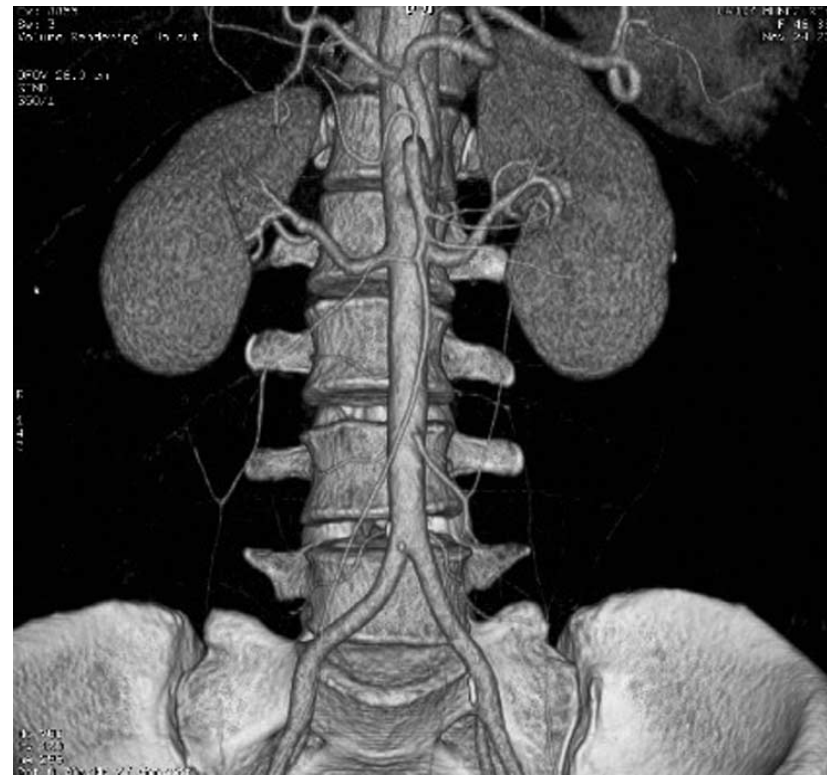

Fig. 2. Arterias renales únicas, originándose a nivel del tercio inferior de L2 y arteria mesentérica superior a nivel de tercio medio de L1.

el otro, a nivel del disco intervertebral entre las vértebras L2 y L3). En el caso restante, la arteria renal derecha tuvo un origen más alto (1/3 medio de L2) que las izquierdas $(1 / 3$ inferior de L2).

\section{DISCUSIÓN}

Las variaciones arteriales adquieren un significado especial cuando se trata de intervenir órganos de diversos sistemas, especialmente cuando se trata de transplantes. El origen y trayecto de los vasos que nutren a los órganos se

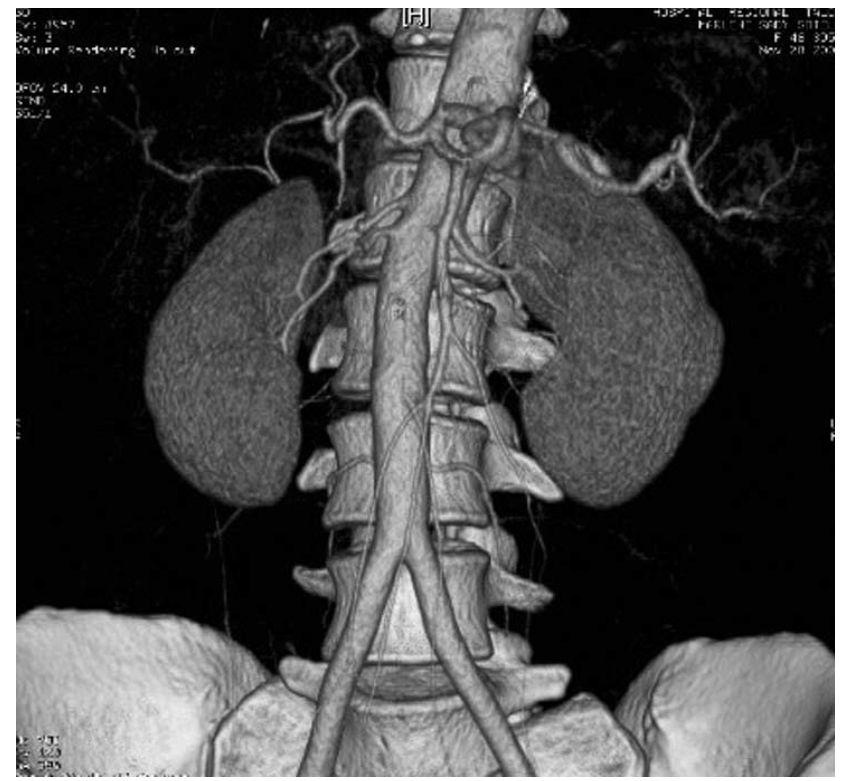

Fig. 3. Arteria renal doble en el lado derecho, originándose a nivel de la vértebra L1.

han determinado clásicamente a través de disecciones anatómicas, repleción de éstos con diversas sustancias que las hacen más visibles entre otras estructuras o a través de rayos X. Actualmente, las imágenes de estructuras anatómicas se pueden visualizar con sofisticados aparatos que logran delimitarlas con alta resolución.

Estudios sobre el origen de las arterias renales han mostrado que éstas se originan por debajo del nivel de origen de la AMS (Testut \& Latarjet, 1969; Williams et al., 1995; Ozan et al., 1997; Moore \& Dalley, 2002; Pennington \& Soames, 2005), destacando Ozan et al. que el ostio de la AR derecha es más craneal que la AR izquierda. 
La literatura es rica en artículos sobre variaciones de origen de las AR, entre las cuales podemos mencionar a la AR derecha originándose desde un tronco común con la arteria mesentérica superior (Dalçik et al., 2000), orígenes altos de la AR derecha (Fernet et al., 1987), presencia de arterias dobles o múltiples (Bulic et al.; Bordei et al., 2004; Loukas et al.; Deepthinath; Tanyeli et al.; Raheem et al., 2007; Olave et al.), origen asociado con otras ramas de la aorta (Bakheit \& Motabagani, 2003). Mención aparte merece la presencia de riñones pélvicos o masas renales que reciben su irrigación de arterias próximas (Dretler et al., 1971; Ohtsuka et al., 1993; Rodríguez et al., 1997).

Las arterias renales y mesentérica superior son ramas de la porción abdominal de la aorta, pudiendo asociar su origen con niveles de la columna vertebral, principalmente las primeras vértebras lumbares.

En el estudio, se pudo observar que la arteria mesentérica superior se origina con frecuencia a nivel de la vértebra L1 y el disco entre las vértebras L1 y L2, considerando que este vaso se presenta único en $100 \%$ de los casos. En general, la literatura informa que la arteria mesentérica superior se origina de la parte anterior de la porción abdominal de la aorta y que irriga el intestino delgado en toda su longitud, excepto la parte superior del duodeno, irrigando también el ciego, colon ascendente y la mitad del colon transverso. Ocasionalmente, la arteria mesentérica superior se puede originar desde el tronco celíaco (Shaeffer, 1942; Testut \& Latarjet; Goss, 1976). Debus et al. (2009) mostraron varias disposiciones de origen de esta arteria, como tronco celiacomesentérico (1-2\%), como tronco lienomesentérico $(0,5 \%)$, como tronco hepatomesentérico (3\%) y como tronco hepatolienomesentérico (1\%), disposiciones no observadas en este estudio.

En nuestro estudio, los niveles de origen de esta arteria respecto a la columna vertebral son variables y en más del $50 \%$ está asociado a la vértebra L1, tanto en su parte superior como en sus partes media e inferior. Sin embargo, encontramos que en $25 \%$ de los pacientes este origen correspondió a la vértebra L2. Por encima de la vértebra L1 o por debajo de la vértebra L2 su origen es menos probable. Sobre esta asociación, origen-vértebras, en la obra de Testut $\&$ Latarjet se señala que su origen estaría situado a nivel del disco entre las vértebras T2 y L1 o también entre las vértebras L2 y L3, disposición muy diferente a nuestros resultados.

En cambio, la arteria renal siendo única bilateralmente en más del $80 \%$ de los casos, se origina entre el tercio medio de la vértebra L1 y el tercio inferior de la vértebra L2. Estos orígenes se visualizan claramente a través de los re- gistros angiográficos. En un estudio por resonancia magnética, Ralston et al. (1992) encontraron que en 75 muestras $(86 \%)$ en que identificaron a la arteria renal derecha (87 de 101 casos), ésta se encuentra muy estrechamente relacionada al disco entre las vértebras L1 y L2. Por su parte, Özkan et al. (2006) determinaron en angiografías de 855 pacientes que el origen de las arterias renales se encuentra de modo general entre el margen superior de la vértebra L1 y el margen inferior de la vértebra L2 en 98\% de los casos, disposición asociada a nuestros resultados, que mostraron esta localización en 90,3\% de los casos en el lado derecho y $87,1 \%$ en el izquierdo.

Especificando un poco más, Özkan et al. señalaron que en el lado derecho, este origen estuvo en relación al margen inferior de la vértebra L1, en $25 \%$ de los pacientes; a nivel del disco entre las vértebras L1 y L2 en 23\% y a nivel del margen superior de la vértebra L2 en $20 \%$. En el lado izquierdo, observaron este origen relacionado con el margen inferior de la vértebra L1 en $23 \%$ de los casos; a nivel del disco entre las vértebras L1 y L2 en $22 \%$ y asociada al margen superior de la vértebra L2 en $24 \%$. Observando y comparando los resultados de ambos lados, las diferencias de origen de las arterias en cuestión fueron mínimas.

Por su parte Lee et al. (2004) identificaron que la porción proximal de la arteria renal derecha se situó en la mitad inferior del cuerpo de la vértebra L1 en 68 de 210 pacientes (32\%), en la mitad superior del cuerpo de esta vértebra en 15 pacientes (7\%), a nivel del disco entre las vértebras L1 y L2 en 109 (52 \%) y en la mitad superior del cuerpo de la vértebra L2 en 17 (8\%).

Nuestros resultados son porcentualmente menores, sin embargo, el origen asociado al disco entre las vértebras L1 y L2 es concordante con los mencionados por Özkan et $a l$. , no así con los de Lee et al. El nivel de origen más alto que encontramos fue a nivel del tercio medio de la vértebra L1, a pesar que Özkan et al. observaron que en alrededor del $25 \%$ se localizó a nivel del margen inferior de esta vértebra. Ocasionalmente se informa de niveles más altos, como el caso en que la arteria renal derecha se originó en relación al disco entre las vértebras T11 y T12 (Fernet et al.) o a nivel del disco entre las vértebras T12 y L1 (Lee et al.). El nivel de origen más bajo observado en nuestro estudio fue a nivel del tercio superior de la vértebra L4, en el lado izquierdo.

De acuerdo a nuestras observaciones y a la división del cuerpo vertebral en tercios, determinamos que los orígenes de las arterias renales de ambos lados eran asimétricos en más del $50 \%$ (14 casos) y de éstos, en el lado derecho era más alto en un poco más de la mitad de los casos. Este por- 
centaje difiere del informado por Ozan et al. cuando indica que el origen de la arteria renal derecha es más craneal que el izquierdo.

Nuestros resultados también mostraron que hubo 5 pacientes $(16,1 \%)$ con más de una arteria renal, resultado más bajo que el $24 \%$ (202 pacientes) encontrado por Özkan et al.

En los pacientes con más de una arteria, 2 de ellos con arterias dobles en el lado derecho, el origen en este lado y en el izquierdo ocurrió al mismo nivel (tercio medio de la vértebra L1). De los 3 que presentaron arterias dobles en el lado izquierdo, en 2 de ellos la arteria renal derecha se originó al mismo nivel que la arteria renal superior izquierda (1/ 3 inferior de la vértebra L2 en uno y disco intervertebral entre las vértebras L2 y L3, en el otro). En el caso restante, la arteria renal derecha tuvo un origen a nivel del cuerpo de la vértebra L2, un poco más alto que las izquierdas.

La determinación de los niveles de origen de las arterias en estudio puede servir de soporte morfológico a los procedimientos quirúrgicos que involucran manejo de órganos en la región lumbar, complementando también el conocimiento arterial de nuestra población.

OLAVE, E.; PUELMA, F.; HENRÍQUEZ, J.; CRUZAT, C. \& SOTO, A. Origin levels of the renal and superior mesenteric arteries in relation to the vertebral column in Chilean individuals. Helicoidal Computed Tomography Study. Int. J. Morphol., 27(2):447-452, 2009.

SUMMARY: The knowledge of the arterial variations is very important for the diagnosis and the surgical procedures. The purpose of this study was determine the number and the originlevels of the renal and superior mesenteric arteries in relation to the vertebral bodies. For this, we used the angiographies of 31 patients, Chileans, adult, of both sexes. The angiographies were obtained by Helicoidal Computed Tomography, in these we localized the origin points of the mentioned arteries. We divided the vertebral bodies in three parts, superior, middle and inferior, considering too the intervertebral disc. In 26 patients, in both sides the renal artery was only; in the right side it was double in 2 patients and in the left side in 3 . The right renal artery originated from the level of L1 vertebra in 11 cases; from the level of L1-L2 intervertebral disc in 7; from the level of the L2 vertebra in 10 and from the level of the L2-L3 intervertebral disc in 2. The left renal artery originated from the level of the L1 vertebra in 9 cases; from the level of the L1-L2 intervertebral disc in 6; from the level of the L2 vertebra in 12 and from the level of the L2-L3 intervertebral disc in 2. The superior mesenteric artery was only in all cases; it origin level was always cranial to the renal arteries origin. The origin level of the superior mesenteric artery was observed in relation to the L1 vertebra in 16 cases and to the level of the L2 vertebra in 8 . We considered specifical origin levels associated to the vertebral body division. The results of this study will complete the knowledge of these arteries and are a contribution to the surgical anatomy of abdominal region.

KEY WORDS: Anatomy; Abdominal aorta; Renal arteries; Superior mesenteric artery.

\section{REFERENCIAS BIBLIOGRAFICAS}

Bakheit, M. A. \& Motabagani, M. A. Anomalies of the renal, phrenic and suprarenal arteries: Case report. East Afr. Med. J., 80(9):497-8, 2003.

Bordei, P.; Sapte, E. \& Iliescu, D. Double renal arteries originating from the aorta. Surg. Radiol. Anat., 26(6):474-9, 2004.

Bulic, K.; Ivkic, G. \& Pavic T. A case of duplicated right renal artery and triplicated left renal artery. Ann. Anat., 178(3):281-3, 1996.

Dalçik, C.; Colak, T.; Ozbeck, A. \& Dalçik, H. Unusual origin of the right renal artery: A case report. Surg. Radiol. Anat.; 22(2):117-8, 2000.

Dhar, P. \& Lal, K. Main and accessory renal arteries-a morphological study. Ital. J. Anat. Embryol., 110(2):101$10,2005$.
Debus, E. S.; Diener, H. \& Larena-Avellaneda, A. Akute intestinale ischämie. Chirurg, 80(4):375-87, 2009.

Deepthinath, R.; Satheesha Nayak, B.; Mehta. R. B.; Bhat, S.; Rodríguez, V.; Samuel, V. P; Venkataramana, V. \& Prasad, A. M. Multiple variations in the paired arteries of the abdominal arteries. Clin. Anat., 19(6):566-9, 2006.

Dretler, S.; Olsson, C. \& Pfister, R. The anatomic, radiologic and clinical characteristics of the pelvic kidney: An analysis of 86 cases. J. Urol., 105:623-7, 1971.

Fernet, M.; Goldlust, D.; Salama, J.; \& Chevrel, J. P. A case of ectopic right renal artery: a radiologic-anatomic variant. Surg. Radiol. Anat., 9(4):319-20, 1987.

Garti, I. \& Meiraz, D. Ectopic origin of main renal artery. Urology, 15(6):627-9, 1980. 
OLAVE, E.; PUELMA, F.; HENRÍQUEZ, J.; CRUZAT, C. \& SOTO, A. Niveles de origen de las arterias renales y mesentérica superior respecto a la columna vertebral en individuos chilenos. Estudio por tomografía computarizada helicoidal. Int. J. Morphol., 27(2):447-452, 2009.

Goss, Ch. M. Gray Anatomía. 29ª ed. Salvat, Barcelona, 1976.

Khamanarong, K; Prachaney, P; Utraravichien, A; Tong-Un T \& Sripaoraya, K. Anatomy of renal arterial supply. Clin. Anat., 17(4):334-6, 2004.

Lee, C. H.; Seo, B. K.; Choi, Y. Ch.; Shin, H. J.; Park, J. H.; Jeon, H. J.; Kim, K. A.; Park, Ch. M. \& Kim, B. H. Using MRI to evaluate anatomic significance of aortic bifurcation, right renal artery, and conus medullaris when locating lumbar vertebral segments. AJR, 182:1295-300, 2004.

Loukas, M.; Aparicio, S.; Beck, A.; Calderón, R. \& Kennedy, M. Rare case of right accessory renal artery originating as a common trunk with the inferior mesenteric artery: a case report. Clin. Anat., 18(7):530-5, 2005.

Moore, K. L. \& Dalley, A. F. Anatomía con Orientación Clínica. $4^{\mathrm{a}}$ Ed. Panamericana, Buenos Aires, 2002.

Ohtsuka, A.; Kikuta, A.; Taguchi, T. \& Murakami, T. Ectopic kidney in front of the right common iliac artery and its blood vascular supply - a case report. Okajimas Folia Anat. Jpn. , 70(1):29-34, 1993.

Olave, E.; Henríquez, J.; Cruzat, C.; Puelma, F. \& Soto, A. Arterias renales múltiples. Int. J. Morphol., 25(4):92730, 2007.

Ozan, H.; Alemdaroglu, A.; Sinav, A. \& Gümüsalan, Y. Location of the ostia of the renal arteries in the aorta. Surg. Radiol. Anat., 19(4):245-7, 1997.

Özkan, U.; Oguskurt, L.; Tercan, F.; Kizilkiliç, O.; Koç, Z. \& Koca, N. Renal artery origins and variations: angiographic evaluation of 855 consecutive patients. Diagn. Interv. Radiol., 12(4):183-6, 2006.

Pennington, N. \& Soames, R. W. The anterior visceral branches of the abdominal aorta and their relationship to the renal arteries. Surg. Radiol. Anat., 27(5):395-403, 2005.

Raheem, O.; Glacken, P.; O’Brien, M.; Hickey, D. \& Mohan, P. A single male cadaver with multiple renal arteries. Ir. J. Med. Sci., 177(3):265-7, 2007.

Ralston, M. D.; Dykes, T. A. \& Applebaum, B. I. Verification of lumbar vertebral bodies. Radiology, 185(2):615-6, 1992.
Rodriguez, C. F. S.; Olave, E.; Gabrielli, C. \& Sousa, L. M. C. Consideraciones anatómicas sobre la fusión renal. Relato de un caso. Rev. Chil. Anat., 15(1):51-5, 1997.

Schaeffer, J. P. Morris' Human Anatomy. $11^{\text {th }}$ ed. New York, McGraw-Hill, 1942.

Tanyeli, E.; Uzel, M. \& Soyluoglu, A. I. Complex renal vascular variation: a case report. Ann. Anat., 188(5):4558, 2006.

Testut, L. \& Latarjet, A. Tratado de Anatomía Humana. Salvat, Barcelona, 1969.

Williams, P. L.; Warwick, R.; Dyson, M. \& Bannister, L. H. Gray Anatomía. 37ª Ed. Guanabara-Koogan, Rio de Janeiro, 1995.

Wozniak, W. T. Origin of the renal arteries from sides of aorta. Folia Morphol. (Warsz), 58(4):259-61, 2000.

Dirección para correspondencia:

Dr. Enrique Olave Riffo

Facultad de Medicina

Universidad de La Frontera

Casilla 54-D Temuco - CHILE

Email: eolave@ufro.cl

Recibido : 12-01-2009

Aceptado: 21-02-2009 